\title{
Coexisting Dementia and Depression in Parkinson's Disease
}

\author{
Mary Sano, PhD; Yaakov Stern, PhD; Janet Williams, DSW; \\ Lucien Coté, MD; Ruth Rosenstein, MPh; Richard Mayeux, MD
}

\begin{abstract}
- Dementia and depression in patients with Parkinson's disease have been reported separately, but their prevalence is controversial. This study examines the coexistence of these two problems and suggests a common underlying biochemical system. We examined these two entities by retrospective chart review and cerebrospinal fluid biochemistry. We found a prevalence of $10.9 \%$ for dementia, $51 \%$ for depression, and $5.4 \%$ for coincident depression and dementia. In a prospective study of patients with Parkinson's disease we found a continuum of cerebrospinal fluid 5-hydroxyindoleacetic acid concentrations. Patients who were either depressed or demented had lower concentrations of this metabolite than other patients with Parkinson's disease, but patients who were depressed and demented had the lowest levels. These results suggest that the coexistence of dementia and depression represents a unique clinical entity in Parkinson's disease. The serotonergic system may be involved in depression and dementia because evidence of a cumulative effect on this biochemical system is present.
\end{abstract}

(Arch Neurol. 1989;46:1284-1286)

$\mathrm{D}^{\mathrm{e}}$ epression and dementia are the most common behavioral disturbances in idiopathic Parkinson's disease (IPD) and each is associated with distinctively different biochemical mechanisms. Dementia has been associated with biologic and pathologic changes in the cholinergic systems.

\footnotetext{
Accepted for publication May 22, 1989.

From the Departments of Neurology (Drs Sano, Mayeux, Stern, Coté, and Rosenstein) and Psychiatry (Drs Mayeux, Stern, and Williams), College of Physicians and Surgeons, Columbia University, New York, NY.

Reprints not available.
}

Choline acetyltransferase activity is lower in demented patients with IPD with and without pathologic changes of Alzheimer's disease (AD) 1 and anticholinergic agents precipitate acute confusional state in demented patients with Parkinson's disease (PD). ${ }^{2}$ Correlations between the severity of dementia and the quantity of neurofibrillary tangles and senile plaques have been reported in $\mathrm{PD},{ }^{3}$ as have decreases in the cholinergic neurons in the basal forebrain. ${ }^{4}$ In addition, some authors have suggested that the serotonergic system is also affected in AD..$^{5}$ 'Amato et $\mathrm{al}^{5}$ reported a decrease in the number of serotonergic neurons in both patients with $A D$ and patients with PD.

Depression in IPD has been related to diminished serotonin, as assessed by decreased concentration of cerebrospinal fluid (CSF) 5-hydroxyindoleacetic acid (5-HIAA). ${ }^{6,7}$ The administration of the serotonin precursor, 5-hydroxytryptophan, may alleviate depression in IPD. This study examined the presence of coexisting depression and dementia in IPD, using retrospective chart review and biochemical studies of CSF.

\section{METHODS \\ Retrospective Chart Review}

Subject Selection.-The medical records of every patient with parkinsonism in an urban hospital seen on all services during an 18-month period were reviewed. The population included private and clinic patients with $90 \%$ receiving annual follow-up. However, only data from the first contact during the study period were included. This review yielded 339 consecutively encountered patients with IPD (78\% inpatient; $22 \%$ outpatient). ${ }^{8}$ The criteria for this diagnosis included the presence of at least two of the four motor manifestations of the disorder (tremor, shuffling gait, bradykinesia, mus- cular rigidity), with a history of insidious onset. Patients with evidence of other known causes of parkinsonism, including postencephalitic, drug-induced, stroke-related, and progressive supranuclear palsy were excluded. Only patients with onset after the age of 40 years were included to ensure that patients had typical IPD.

Data Extraction.-A systematic data collection form to extract clinical information from records was designed by us prior to the survey. The first encounter was used to estimate the prevalence of dementia and depression and any follow-up entries were used to substantiate the initial impressions. Consensus on criteria for the diagnoses of depression and dementia was reached prior to the study, and interrater agreement was found to be high. ${ }^{8}$

Diagnoses. - Research diagnostic criteria for primary degenerative dementia and the criteria for major depression were based on Diagnostic and Statistical Manual of Mental Disorders (DSM-III).$^{9}$ Dementia is sustained impairment in intellectual functioning that was not considered drug related and without an alteration in consciousness. A written description of mental status was required and it had to include evidence of impairment in memory, orientation, and general knowledge. The details of this method are based on the findings of Rajput et al ${ }^{10}$ with modifications that have been previously reported. ${ }^{8}$

The presence of depression was assessed at the time of the study and excluded a history of affective disorder. Depression is a sustained dysphoric mood characterized by feelings of sadness and associated with changes in appetite and sleep habits. Fleeting or slight mood changes were excluded.

Results.-Using the criteria described, we found that 37 patients $(10.9 \%)$ were demented and $172(51 \%)$ were depressed. Seventeen patients $(5.4 \%)$ had depression and dementia (Table 1). The demented patients and those with dementia and depression were significantly older and had later onset of PD symptoms than the other patients. In addition this group had higher scores on the PD Rating Scale, suggesting more severe disease. The rate of dementia is signifi- 


\begin{tabular}{|ccccc|}
\hline \multicolumn{2}{|c|}{ Table 1.-Description of Groups in the Chart Review Study $(\mathrm{N}=339)^{*}$} \\
\hline & $\begin{array}{c}\text { Not Depressed, } \\
\text { Not Demented } \\
(\mathbf{n}=\mathbf{1 7 7})\end{array}$ & $\begin{array}{c}\text { Demented } \\
(\mathbf{n}=\mathbf{1 7})\end{array}$ & $\begin{array}{c}\text { Depressed } \\
(\mathbf{n}=\mathbf{1 3 3})\end{array}$ & $\begin{array}{c}\text { Depressed and } \\
\text { Demented } \\
(\mathbf{n}=\mathbf{1 2})\end{array}$ \\
\hline Age, $\dagger \mathrm{y}$ & $67.02 \pm 9.0(\mathrm{a}) \neq$ & $76.29 \pm 6.9(\mathrm{~b}) \neq$ & $66.84 \pm 8.8(\mathrm{a})$ & $74.53 \pm 8.1(\mathrm{~b})$ \\
\hline Duration, $y$ & $9.05 \pm 7.1$ & $9.06 \pm 7.2$ & $7.89 \pm 4.5$ & $8.27 \pm 4.4$ \\
\hline $\begin{array}{c}\text { Years of levodopa } \\
\text { treatment }\end{array}$ & $5.58 \pm 4.5$ & $5.97 \pm 4.8$ & $6.35 \pm 4.4$ & $4.42 \pm 3.6$ \\
\hline Age of onset, $\dagger y$ & $59.37 \pm 9.8(\mathrm{a})$ & $67.32 \pm 10.3(\mathrm{~b})$ & $59.08 \pm 9.6(\mathrm{a})$ & $66.33 \pm 9.7(\mathrm{~b})$ \\
\hline $\begin{array}{c}\text { Symptom } \\
\text { severity } \dagger\end{array}$ & $26.18 \pm 17.3(\mathrm{a})$ & $50.39 \pm 17.7(\mathrm{~b})$ & $37.91 \pm 17.0(\mathrm{a})$ & $50.25 \pm 22.1(\mathrm{~b})$ \\
\hline
\end{tabular}

"Values are means \pm SDs.

$\dagger P<.001$ for analyses of variance comparing all group means for each variable.

¥Means and SDs with the same letters $(a, b)$ do not differ significantly in post hoc analyses.

Table 2.-Demographic Variables and Cerebrospinal Fluid Biogenic Amine Metabolites in Patients With Parkinson's Disease $(n=110)^{*}$

\begin{tabular}{|lcccc|}
\hline & $\begin{array}{c}\text { Not Depressed } \\
\text { or Demented } \\
\text { (n=46) }\end{array}$ & $\begin{array}{c}\text { Demented } \\
(\mathbf{n}=\mathbf{3 1})\end{array}$ & $\begin{array}{c}\text { Depressed } \\
(\mathbf{n}=\mathbf{2 7})\end{array}$ & $\begin{array}{c}\text { Depressed and } \\
\text { Demented } \\
(\mathbf{n}=\mathbf{6})\end{array}$ \\
\hline Age, $\mathrm{y}$ & $63.4 \pm 9.3(\mathrm{bc})$ & $72.4 \pm 7.4(\mathrm{a})$ & $62.7 \pm 8.0(\mathrm{c})$ & $69.6 \pm 12.8+(\mathrm{ab})$ \\
\hline Education, $\mathrm{y}$ & $12.8 \pm 3.7$ & $14.0 \pm 4.7$ & $13.9 \pm 3.9$ & $14.0 \pm 2.5$ \\
\hline Duration, $\mathrm{y}$ & $7.5 \pm 5.5$ & $7.9 \pm 5.7$ & $6.6 \pm 4.2$ & $10.9 \pm 13.1$ \\
\hline $\mathrm{mMMS} \mathrm{score}$ & $50.2 \pm 6.5(\mathrm{a})$ & $34.9 \pm 10.2(\mathrm{~b})$ & $50.7 \pm 4.6(\mathrm{a})$ & $35.4 \pm 8.7 \neq(\mathrm{b})$ \\
\hline $\mathrm{HVA}, \mathrm{ng} / \mathrm{mL}$ & $45.2 \pm 40.7$ & $40.6 \pm 27.4$ & $49.1 \pm 43.1$ & $31.2 \pm 18.8$ \\
\hline $\mathrm{MHPG}, \mathrm{ng} / \mathrm{mL}$ & $11.4 \pm 6.9$ & $8.0 \pm 4.7$ & $9.0 \pm 5.1$ & $9.0 \pm 5.0$ \\
\hline $5 \cdot \mathrm{HIAA}, \mathrm{ng} / \mathrm{mL}$ & $20.9 \pm 7.9(\mathrm{a})$ & $15.8 \pm 6.3(\mathrm{ab})$ & $15.9 \pm 6.1(\mathrm{ab})$ & $12.4 \pm 4.8 \neq(\mathrm{b})$ \\
\hline
\end{tabular}

*Values are means \pm SDs. Means and SDs with the same letters $(a, b, c)$ do not differ significantly in post hoc analysis. mMMS indicates modified Mini-Mental State Exam; HVA, homovanillic acid; MHPG, 3-methoxyl4-hydroxy-phenylglycol; and 5-HIAA, 5-hydroxyindoleacetic acid.

$+P<.001$ for analyses of variance (ANOVAs) comparing all group means for each variable

$\ddagger P<.05$ for ANOVAs comparing all group means for each variable.

cantly higher than would be predicted; it is 3.8 times greater than expected when compared with a population study that used volunteers older than 60 years. ${ }^{11}$

\section{Prospective Inpatient Studies}

Subjects.-Over 5 years, 115 patients with IPD encountered within the hospital or outpatient services and giving informed consent prior to hospital admission were included. They participated in one of two inpatient research protocols. In the initial protocol, patients were taken off all treatment with dopamine agonist medications before completing the psychiatric, neuropsychologic, and CSF measures. ${ }^{5}$ In a later protocol there was no medication withdrawal and all procedures were completed while patients were receiving optimal doses of levodopa (L-dopa) or a combination of - carbidopa and levodopa (Sinemet). ${ }^{6}$ Since the groups were comparable, they were combined for this report.

For comparison purposes, a group of patients $(\mathrm{N}=25)$ who met National Institute of Neurological Communicative Disorders and Stroke-Alzheimer's Disease and Related Disorders Association criteria for probable $\mathrm{AD}$ were included. These patients were only mildly impaired and were not receiving medication at the time of the study. They were free of affective complaints and participated in the neuropsychologic and CSF studies described below.

Procedures.-Psychiatric Diagnosis.The Structured Clinical Interview was com- pleted with each patient by a trained psychiatric interviewer. A diagnosis was derived according to DSM-III criteria. ${ }^{9}$ When needed, a family member was also interviewed to provide additional information. The Hamilton Rating Scale of Depression was also administered.

Neuropsychologic Studies._Each patient received a battery of neuropsychologic tests measuring memory, attention, language, constructional ability, and general cognition. These studies were used to confirm the diagnosis of dementia according to $D S M-I I I$ criteria.

CSF Studies.-A lumbar puncture for CSF was performed after 18 hours of bed rest. Cerebrospinal fluid, $20 \mathrm{~mL}$, was removed and the metabolites of dopamine (homovanillic acid), serotonin (5HIAA), and norepinephrine 3-methoxyl4-hydroxy-phenylglycol (MHPG) were measured using high-performance liquid chromatography with electrochemical detection. ${ }^{12}$

\section{RESULTS}

Four groups of patients with IPD were identified: demented ( $n=31$ ), depressed $(n=27)$, both demented and depressed $(n=6)$, or unaffected $(\mathrm{n}=46)$ (Table 2). As in the chart review study, the demented patients were significantly older than the nondemented patients. However, those who were depressed and demented
Table 3.-Comparison of

Demographic Data and Cerebrospinal Fluid Biogenic Amine Metabolites in Patients With Parkinson's Disease and Alzheimer's Disease $(n=135)^{*}$

\begin{tabular}{|lcc|}
\hline & $\begin{array}{c}\text { Alzheimer's } \\
\text { Disease } \\
\text { (n= 25) }\end{array}$ & $\begin{array}{c}\text { Parkinson's } \\
\text { Disease } \\
\text { (n=110) }\end{array}$ \\
\hline Age, y & $68.1 \pm 9.3$ & $66.1 \pm 9.5$ \\
$\mathrm{mMMS}$ score & $39.6 \pm 9.9$ & $45.5 \pm 10.2$ \\
$\mathrm{MHPG}, \mathrm{ng} / \mathrm{mL}$ & $4.23 \pm 2.9$ & $3.82 \pm 2.7$ \\
HVA, ng $/ \mathrm{mL}$ & $49.06 \pm 47.5$ & $44.0 \pm 36.9$ \\
5-HIAA, ng/mL & $28.5 \pm 20.4$ & $17.7 \pm 7.4$ \\
\hline
\end{tabular}

*Values are means \pm SDs. See Table 2 asterisk footnote for explanation of abbreviations.

were not older than the other groups. There were no other significant age differences among groups. The demented and the demented and depressed groups had significantly lower modified Mini-Mental State Examination scores.

Patients with depression and dementia had significantly lower CSF 5HIAA levels than the unaffected group with PD (Table 2). Patients who were (only) depressed or (only) demented had CSF 5-HIAA levels that were lower than the unaffected group with PD and higher than the demented and depressed group. However, these differences were not significant. There was no significant difference among the groups in homovanillic acid or MHPG.

Although demented patients were significantly older, age did not account for the reduction in CSF 5-HIAA values; the correlation between age and CSF 5-HIAA was not significant $(r=$ $-.037)$.

Table 3 summarizes comparisons between patients with AD and IPD. The groups did not differ in age. The group with $\mathrm{AD}$ had significantly higher levels of CSF 5-HIAA than the patients with IPD, a finding we have reported previously.? Performance on the modified Mini-Mental State Examination, a brief test of mental status, did not differ in these two patient groups, which is expected since the group with IPD includes demented patients.

\section{COMMENT}

The present studies suggest that depression and dementia in IPD may have a common biologic mechanism. The demented and depressed patients had significantly lower levels of the serotonin metabolite, a finding that has been reported in depressed patients with PD by $\mathrm{us}^{6,7}$ and by others. ${ }^{13}$ It is unlikely that the association between dementia and the serotonergic 
system is an artifact of the pseudodementia of affective illnesses ${ }^{14}$ since lowered 5-HIAA levels were also present in demented patients who were not depressed. Patients with depression and dementia had lower levels of the serotonergic metabolite than those patients with either entity alone. Although this difference was not significant, it may suggest an additive effect of the two phenomena.

Much overlap exists between dementia in $P D$ and AD. Neurofibrillary plaques and tangles, the hallmark of $A D$, have been identified in patients with PD. ${ }^{1}$ There are also biochemical similarities between PD and AD. Levels of CSF 5-HIAA can be reduced in patients with $A D,{ }^{5}$ although we found that the CSF 5-HIAA levels in patients with $A D$ were not as low as in patients with PD.

In the chart review, prevalence of dementia was significantly higher than reported in an age-matched comparison group. Dementia was associated with patients with more severe parkinsonism and older age of onset than nondemented patients with PD. Depression in PD was frequent in the retrospective and the prospective studies, although the actual prevalence differed. Sampling and assessment techniques may account for much of this variation, with a diagnosis based on the presence of symptoms of depression in the chart review yielding higher prevalence than diagnoses made by direct interview. The depressed patients did not differ in age and severity from the unaffected patients with PD. However, the depressed group was younger than the group with depression and dementia. Perhaps depression is an early complication of PD. One might speculate that the occurrence of depression in PD would increase the probability for dementia later.

The coexistence of depression and dementia has been examined in other populations. Kay et al ${ }^{15}$ found no association between the diagnosis of depression and dementia in a sample of community dwelling elderly. Others have suggested that depression may be an antecedent to dementia. In a clinicbased population, referred for cognitive problems, more than half of the patients initially diagnosed as being depressed and not demented were found to be demented at 3-year follow- up assessment. Age was the only variable that was associated with the demented group, making it difficult to determine the true impact of depression. ${ }^{16}$ While the involvement of a common biochemical system suggests an association between these entities, only a prospective study can determine if depression increases the risk of dementia in PD.

In summary, the finding of lowered serotonin metabolite levels in both depressed and demented patients suggests a common biochemical mechanism for both of these behavioral manifestations of PD. Coexisting dementia and depression in PD may result from a widespread degeneration of dopaminergic, serotonergic, and presumably cholinergic neurons.

This investigation was supported in part by Health Services grant AG-02802 from the National Institute on Aging, National Institutes of Health, Bethesda, Md; and the Parkinson's Disease Foundation. It was completed in a federally supported General Clinical Research Center and the data were stored and analyzed on CLINFO (RR00645). Dr Sano is a Herbert Irving assistant professor in the Department of Neurology, College of Physicians and Surgeons of Columbia University and Presbyterian Hospital of New York, NY.

\section{References}

1. Perry RH, Tomlinson BE, Candy JM, et al. Cortical cholinergic deficit in mentally impaired parkinsonian patients. Lancet. 1983;1:789-790.

2. Desmet Y, Ruberg M, Serdara M, Dubois B, Lhermitte F, Agid Y. Confusion, dementia, and anticholinergics in Parkinson's disease. $J$ Neurol Neurosurg Psychiatry. 1982;45:1161-1164.

3. Boller F, Mizutani T, Roessmann U, Gambetti P. Parkinson's disease, dementia, and $\mathrm{Alz}$ heimer's disease: clinicopathological correlations. Ann Neurol. 1980;1:329-335.

4. Whitehouse P, Hegreen JC, White C, Price DL. Basal forebrain neurons in the dementia of Parkinson's disease. Ann Neurol. 1983;13:243-248.

5. D'A mato RA, Zweig RM, Whitehouse PJ, et al. Aminergic systems in Alzheimer's disease and Parkinson's disease. Ann Neurol. 1987;22:229-236.

6. Mayeux R, Stern Y Coté L. Williams JBW. Altered serotonin metabolism in depressed patients with Parkinson's disease. Neurology.
1984;34:642-646.

7. Mayeux R, Stern Y, Sano M, Williams JBW, Coté L. Relationship of serotonin to depression in Parkinson's disease. Movement Disord. 1988;3: 237-244.

8. Mayeux R, Stern $\mathrm{Y}$, Rosenstein $\mathrm{R}$, et al. An estimate of the prevalence of dementia in idiopathic Parkinson's disease. Arch Neurol. 1988; 45:260-262.

9. American Psychiatric Association, Committee on Nomenclature and Statistics. Diagnostic and Statistical Manual of Mental Disorders, Third Edition. Washington, DC: American Psychiatric Association; 1980:205-224

10. Rajput AH, Offord KP, Beard CM, Kurland LT. A case-control study of smoking habits, de mentia, and other illnesses in idiopathic Parkin son's disease. Neurology. 1987;29:1253-1260.

11. Sayetta RB. Rates of senile dementiaAlzheimer's type in the Baltimore longitudinal study. J Chem Dis. 1986;39:271-286.

12. Mayeux R, Stern Y, Sano M, Coté I, Williams JBW. Clinical and biochemical correlates of bradyphrenia in Parkinson's disease. Neurology. 1987;37:1130-1134.

13. Kostic VS, Djuricic BM, Čovičković-Šternić N, Bumbaširević L, Nikolić M, Mršulja BB. Depression and Parkinson's disease: possible role of serotonergic mechanisms. J Neurol. 1987;12:9496.

14. Taylor A, Saint-Cyr JA, Lang AE. Dementia prevalence in Parkinson's disease. Lancet. 1985;1:1037.

15. Kay D, Henderson A, Scott R, Wilson J, Rickwood D, Grayson D. Dementia and depression among the elderly living in Hobart community. Psychol Med. 1985;15:771-788.

16. Reding M, Haycox J, Blass J. Depression in patients referred to a dementia clinic. $\mathrm{Arch}$ Neu rol. 1985;42:894-896. 